\title{
Reconstruction of metabolic pathway for isobutanol production in Escherichia coli
}

\author{
Shuhei Noda ${ }^{1}$, Yutaro Mori ${ }^{1}$, Sachiko Oyama ${ }^{1}$, Akihiko Kondo ${ }^{1,2}$, Michihiro Araki ${ }^{3}$ and Tomokazu Shirai ${ }^{*}$
}

\begin{abstract}
Background: The microbial production of useful fuels and chemicals has been widely studied. In several cases, glucose is used as the raw material, and almost all microbes adopt the Embden-Meyerhof (EM) pathway to degrade glucose into compounds of interest. Recently, the Entner-Doudoroff (ED) pathway has been gaining attention as an alternative strategy for microbial production.

Results: In the present study, we attempted to apply the ED pathway for isobutanol production in Escherichia coli because of the complete redox balance involved. First, we generated ED pathway-dependent isobutanol-producing E. coli. Thereafter, the inactivation of the genes concerning organic acids as the byproducts was performed to improve the carbon flux to isobutanol from glucose. Finally, the expression of the genes concerning the ED pathway was modified.
\end{abstract}

Conclusions: The optimized isobutanol-producing E. coli produced $15.0 \mathrm{~g} / \mathrm{L}$ of isobutanol as the final titer, and the yield from glucose was $0.37 \mathrm{~g} / \mathrm{g}$ (g-glucose/g-isobutanol).

Keywords: Entner-Doudoroff pathway, Escherichia coli, Glucose, Isobutanol, Microbial catalysis

\section{Background}

The microbial production of valuable compounds, such as chemicals, fuels, and drugs, has been widely researched for the last few decades. Using genetically and metabolically engineered microorganisms as the host strain, these useful compounds were produced mainly from glucose as the raw material [1-6]. In nature, almost all microbes follow the Embden-Meyerhof (EM) pathway as the central metabolism system to acquire energy from carbohydrates such as glucose. In the EM pathway, $1 \mathrm{~mol}$ of glucose is metabolized to $2 \mathrm{~mol}$ of pyruvate via 10 -step reactions. In the process, particularly bacteria can acquire $2 \mathrm{~mol}$ of adenosine triphosphate (ATP) and 2 mol of reduced nicotinamide adenine dinucleotide (NADH). E. coli and other bacteria follow an alternative metabolic pathway called as the Entner-Doudoroff (ED) pathway. In this pathway, similar to the EM pathway, $1 \mathrm{~mol}$ of glucose is metabolized to $2 \mathrm{~mol}$ of pyruvate; however, this pathway

\footnotetext{
*Correspondence: tomokazu.shirai@riken.jp

${ }^{1}$ Center for Sustainable Resource Science, RIKEN, 1-7-22, Suehiro-cho,

Tsurumi-ku, Yokohama, Kanagawa 230-0045, Japan

Full list of author information is available at the end of the article
}

involved only 4 enzymes. Using the ED pathway, bacteria can acquire $1 \mathrm{~mol}$ of ATP, NADH, and reduced NADH phosphate (NADPH) [7].

Some reports concerning microbial production of valuable chemicals using the ED pathway are available. $\mathrm{Ng}$ et al. have introduced a synthetic ED pathway from Zymomonas mobilis into E. coli to produce terpenoid [8]. Zhao et al. have reported ethanol production from lignocellulosic biomass using $Z$. mobilis as the host strain, which originally adopted ED pathway to acquire energy from carbohydrates [9]. In various microorganisms, researches regarding applying the ED pathway for bioproduction have been widely undertaken [10-13].

Isobutanol, one of the most attractive biofuels, has been widely studied for industrial production. A synthetic pathway of isobutanol has previously been constructed, and the suitable genes were isolated $[14,15]$. This synthetic pathway initiates from the aldol condensation of 2 mol of pyruvate to 2-acetolactate by acetolactate synthase (AlsS) from Bacillus subtilis. In addition, the overexpression of the enzymes ketol-acid reductoisomerase (IlvC) and dihydroxy-acid dehydratase (IlvD) from E. coli and 2-ketoisovalerate decarboxylase (Kivd) and alcohol 
dehydrogenase (AdhA) from Lactococcus lactis contribute to the synthesis of isobutanol [14]. In this synthetic pathway, $1 \mathrm{~mol}$ of NADH and NADPH were consumed (Fig. 1). In the ED pathway of E. coli, $1 \mathrm{~mol}$ of NADH and NADPH were produced from 1 mol of glucose, as shown above. Therefore, the combination of ED pathway and the isobutanol synthetic pathway is expected to be one of the powerful strategies to achieve high isobutanol production because of the complete redox balance (Fig. 1). Although isobutanol has reportedly been produced using E. coli carrying a synthetic ED pathway of $Z$. mobilis [16], the EM pathway and pentose phosphate pathway (PPP) has remained in the metabolic pathway of $E$. coli used in the report; this indicates that the metabolic pathway for isobutanol production involving the ED pathway should be improved from the perspective of redox balance.

In the present study, we performed isobutanol production using genetically modified $E$. coli available for the ED pathway as the host strain. For this purpose, we generated $E$. coli strain strictly available for the ED pathway by inactivating the genes encoding glucose-6-phosphate isomerase (Pgi) and 6-phosphogluconate dehydrogenase (Gnd) passing to the EM pathway and PPP, respectively. Thereafter, the pathways to produce byproducts, such as organic acids, were interrupted from the metabolic pathway of the E. coli strain available for the ED pathway. Finally, the ED pathway was modified by overexpressing the genes concerning the endogenous ED pathway in E. coli. Using the E. coli transformant thus generated, we successfully demonstrated isobutanol production. The redox balance in the metabolic pathway is one of the most important factors, and the use of ED pathway would be a promising tool for microbial production.

\section{Results}

\section{Creation of E. coli strain available for the ED pathway}

To create isobutanol-producing $E$. coli with the complete redox balance, we attempted to modify the metabolic pathway of E. coli K-12 MG1655 (called as CFTi2 in the present study). First, we inactivated pgi to prevent the carbon flux to the EM pathway (CFTi3). To activate the ED pathway, gntR-encoding DNA-binding transcriptional repressor was interrupted. Although the CFTi4 strain thus generated was available for the ED pathway, the carbon flux to PPP pathway had already remained and the redox balance was unsuitable for isobutanol production. To overcome this difficulty, gnd-encoding 6-phosphogluconate dehydrogenase was removed from CFTi4 strain and the completely ED pathway-dependent E. coli was constructed (CFTi5) (Fig. 1). Here, trace experiments with $\left[1{ }^{13} \mathrm{C}\right]$ glucose were performed to verify that the constructed strain metabolized glucose using the ED pathway (Additional file 1: Fig. S1). By analyzing mass isotopomer distributions of TBDMS-derivatized Ala, it was confirmed (Additional file 1: Table S2). The strains constructed in the present study are shown in Table 1 .

The synthetic gene clusters concerning isobutanol production were introduced into the $E$. coli strains generated above. Using these isobutanol-producing $E$. coli transformants, isobutanol production was evaluated under aerobic condition. Figure 2 shows the CFTi21, CFTi31, CFTi41, and CFTi51 culture profiles. The highest amount of isobutanol produced was $11.8 \mathrm{~g} / \mathrm{L}$ in CFTi51 culture, which is a completely ED pathwaydependent strain, following 48 -h cultivation, whereas CFTi51 is the unique strain that completely consumed glucose, its maximal cell growth was the lowest among the 4 transformants used in the present experiment. Although CFTi41 showed the highest cell growth, the amount of isobutanol produced in its culture was lowest among 4 strains. Moreover, we measured the amount of organic acids produced as byproducts. The ED pathway-dependent CFTi51 strain produced $1.5 \mathrm{~g} / \mathrm{L}$ of acetate until 48-h cultivation, whereas the amount of the byproduct produced by CFTi21 that adopted the EM pathway was almost $3.0 \mathrm{~g} / \mathrm{L}$ (Additional file 1: Fig. S2). The titer of isobutanol, yield from glucose, and rate of glucose uptake are summarized in Additional file 1: Table S3. The yield of isobutanol from glucose in CFTi51 after $48 \mathrm{~h}$ of cultivation was $0.24 \mathrm{~g} / \mathrm{g}$ (g-isobutanol/g-glucose).

\footnotetext{
(See figure on next page.)

Fig. 1 The synthetic metabolic pathway for isobutanol production in E. coli. PEP, phosphoenolpyruvate; AcCoA, acetyl coenzyme A; Pyr, pyruvate; $\mathrm{NADPH}$, reduced nicotinamide adenine dinucleotide phosphate; $\mathrm{NADP}^{+}$, oxidised $\mathrm{NADPH}$; $\mathrm{NADH}$, reduced nicotinamide adenine dinucleotide; $N A D^{+}$, oxidised NADH; G6P, glucose 6-phosphate; F6P, fructose 6-phosphate; FBP, fructose 1,6-bisphosphate; GAP, glyceraldehyde 3-phosphate; DHAP, dihydroxyacetone phosphate; PGA, glycerate 3-phosphate; 6PGL, 6-phospho-D-glucono-1,5-lactone; 6PGC, 6-phospho-D-gluconate; 2ddg6p, 2-dehydro-3-deoxy-D-gluconate 6-phosphate; Ru5P, D-ribulose 5-phosphate; R5P, D-ribose 5-phosphate; Xu5P, D-xylulose 5-phosphate; S7P, D-sedoheptulose 7-phosphate; E4P, D-erythrose 4-phosphate; pgi, glucose-6-phosphate isomerase; gntR, DNA-binding transcriptional repressor; gnd, 6-phosphogluconate dehydrogenase; pflB, pyruvate formate lyase; IdhA, D-lactate dehydrogenase; pta, phosphate acetyltransferase; zwf, glucose-6-phosphate dehydrogenase; pgl, 6-phosphogluconolactonase; edd, phosphogluconate dehydratase; eda, KHG/KDPG aldolase; ackA, acetate kinase; alsS, acetolactate synthase; ilvC, ketol-acid reductoisomerase; ilvD, dihydroxy-acid dehydratase; kivd, 2-ketoisovalerate decarboxylase; adhA, alcohol dehydrogenase
} 


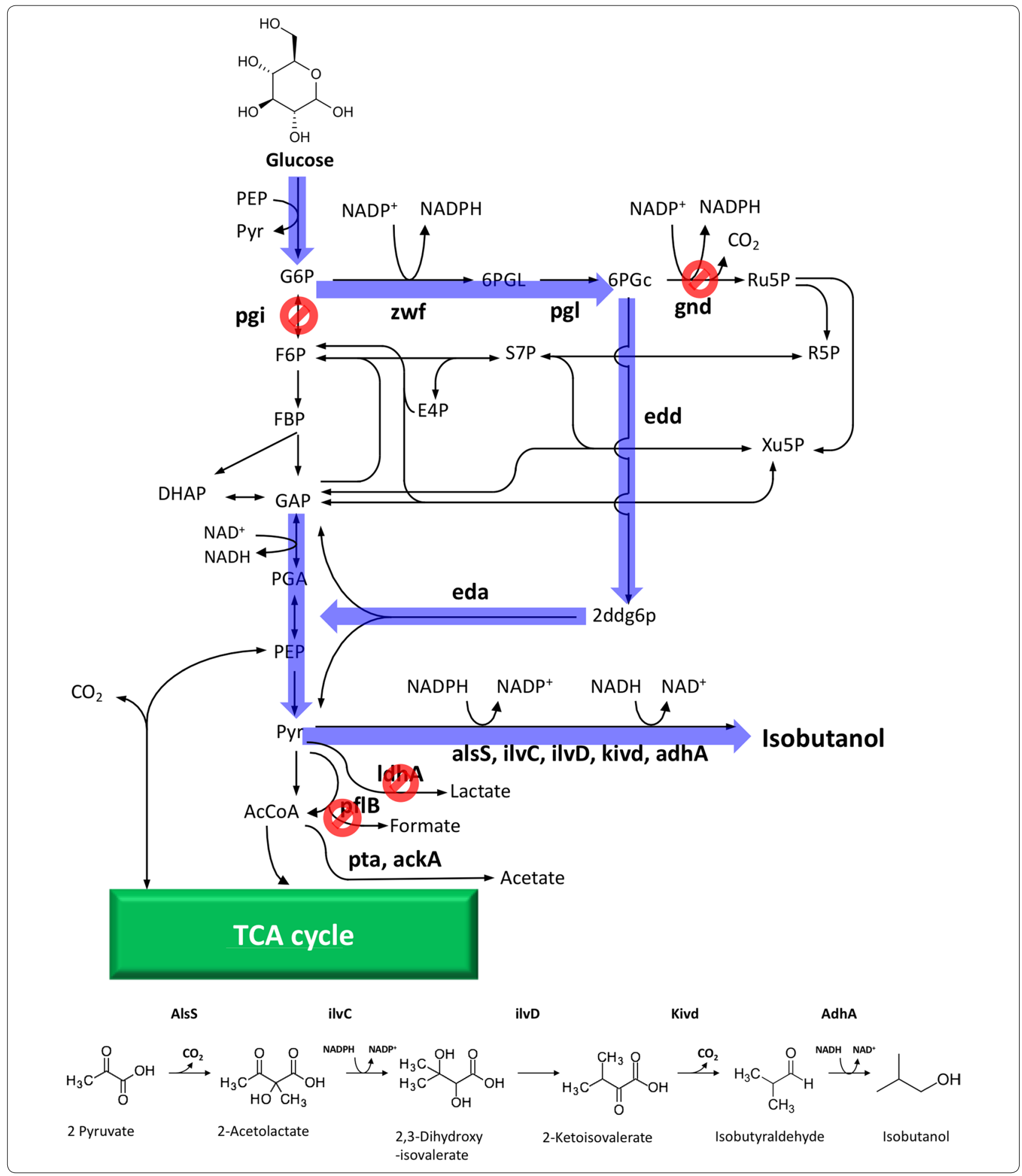

Inactivation of the pathway to produce byproducts in $E$. coli

Furthermore, to prevent volatilization of isobutanol, test tubes used for cultivation were screw-capped after $18 \mathrm{~h}$ of cultivation. In this case, cultures were assumed to be relatively under anaerobic condition after $18 \mathrm{~h}$ of cultivation. In E. coli culture, under anaerobic condition, yielding organic acids, such as lactate, was possible. To further 
Table 1 Strains, plasmids, and transformants used in the present study

\begin{tabular}{|c|c|c|}
\hline Strains or plasmids & Genotype & Source or reference \\
\hline \multicolumn{3}{|l|}{ Strains } \\
\hline NovaBlue & $\begin{array}{l}\text { endA1 hsdR17 (rK12- mK12+) supE44 thi-l gyrA96 relA1 lac recA1/F' } \\
{\left[\text { proAB }^{+} \text {lacl } 19 \mathrm{Z} \triangle \mathrm{M} 15:: \operatorname{Tn} 10 \text { (Tet')]; used for gene cloning }\right.}\end{array}$ & Novagen \\
\hline ATCC 700926 & MG1655 & American Type Culture Collection \\
\hline CFTi2 & ATCC 700926 & ATCC \\
\hline CFTi3 & CFTi2 $\triangle p g i$ & This study \\
\hline CFTi4 & CFTi3 $\triangle g n t R$ & This study \\
\hline CFTi5 & CFTi4 $\triangle$ gnd & This study \\
\hline CFTi6 & CFTi5 $\triangle p f l B$ & This study \\
\hline CFTi9 & CFTi6 $\triangle l d h A$ & This study \\
\hline CFTi10 & CFTi7 $\Delta p t a$ & This study \\
\hline CFTi2a & CFTi2 harboring pTka & This study \\
\hline CFTi3a & CFTi3 harboring pTka & This study \\
\hline CFTi4a & CFTi4 harboring pTka & This study \\
\hline CFTi5a & CFTi5 harboring pTka & This study \\
\hline CFTi6a & CFTi6 harboring pTka & This study \\
\hline CFTi9a & CFTi9 harboring pTka & This study \\
\hline CFTi10a & CFTi10 harboring pTka & This study \\
\hline CFTi21 & CFTi2a harboring p23SCD & This study \\
\hline CFTi31 & CFTi3a harboring p23SCD & This study \\
\hline CFTi41 & CFTi4a harboring p23SCD & This study \\
\hline CFTi51 & CFTi5a harboring p23SCD & This study \\
\hline CFTi61 & CFTi6a harboring p23SCD & This study \\
\hline CFTi91 & CFTi9a harboring p23SCD & This study \\
\hline CFTi101 & CFTi10a harboring p23SCD & This study \\
\hline CFTi91co & CFTi91 harboring pSAK & This study \\
\hline CFTi91zp & CFTi91 harboring pSzp & This study \\
\hline CFTi91zpee & CFTi91 harboring pSzpee & This study \\
\hline \multicolumn{3}{|l|}{ Plasmids } \\
\hline pTrcHis B & $P_{\text {tro, }}$ pBR322 ori, Amp ${ }^{r}$ & Life Technologies \\
\hline pZE12MCS & $\mathrm{P}_{\text {LlacO1}}$, colE1 ori, $\mathrm{Amp}^{\mathrm{r}}$ & Expressys \\
\hline pZA23MCS & $\mathrm{P}_{\text {AlacO1}}, \mathrm{P} 15 \mathrm{~A}$ ori, $\mathrm{Km}^{\mathrm{r}}$ & Expressys \\
\hline pZA23trc & $P_{\text {trc }}$ P15A ori, $\mathrm{Km}^{r}$ & This study \\
\hline pSAK & $\mathrm{P}_{\text {Alaco1, }}$ SC101 ori, $\mathrm{Cm}^{r}$ & {$[23]$} \\
\hline pZka & pZE12MCS containing kivd and adhAfrom Lactococcus lactis & This study \\
\hline pTka & pTrcHis B containing kivd and adhAfrom Lactococcus lactis & This study \\
\hline p23S & pZA23trc containing alsS from B. subtilis & This study \\
\hline p23SCD & pZA23trc containing alsS from B. subtilis, ilvC and ilvD from Escherichia coli & This study \\
\hline pSp & pSAK containing pg/ from E. coli & \\
\hline pSzp & pSAK containing zwf and pgl from E. coli & This study \\
\hline pSzpee & pSzp containing edd and eda from E. coli & This study \\
\hline
\end{tabular}

Amp, ampicillin; Km, kanamycin; $\mathrm{Cm}$, chloramphenicolp

encourage isobutanol production, we attempted to disrupt the carbon flux to some organic aids as byproducts. Some organic acids, such as acetate, lactate, and formate are produced via pyruvate and acetyl coenzyme A (acetylCoA) in E. coli. Using CFTi5 as the base strain, pflB, $l d h A$, and $p t a$ (encoding pyruvate formate lyase, D-lactate dehydrogenase, and phosphate acetyltransferase, respectively) were sequentially inactivated (Fig. 1).

Using the modified isobutanol-producing E. coli, we performed isobutanol production. Figure 3 shows the CFTi61, CFTi91, and CFTi101 culture profiles. 

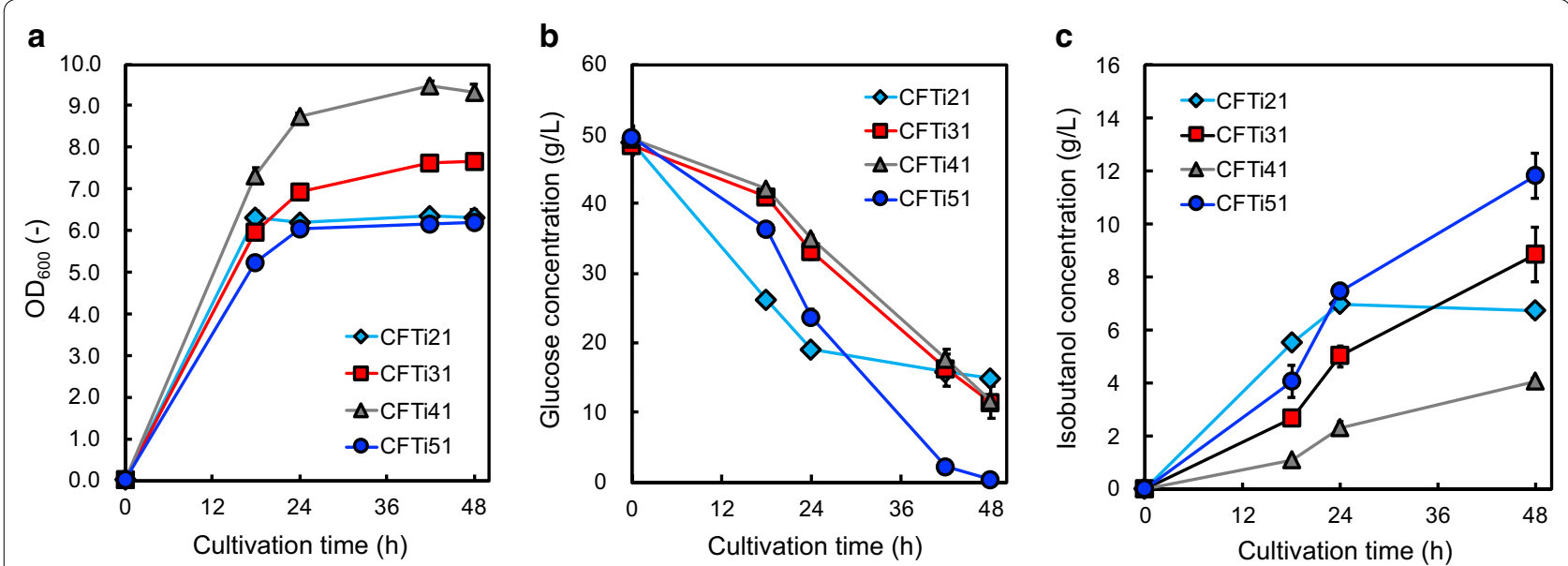

Fig. 2 CFTi21, CFTi31, CFTi41, and CFTi51 culture profiles. Time courses of a bacterial cell growth and $\mathbf{b}$ glucose consumption. cThe amount of isobutanol produced in CFTi21 (diamonds), CFTi31 (squares), CFTi41 (triangles), and CFTi51 (circles) cultures under aerobic condition. Data are presented as the mean \pm standard deviation of three independent experiments
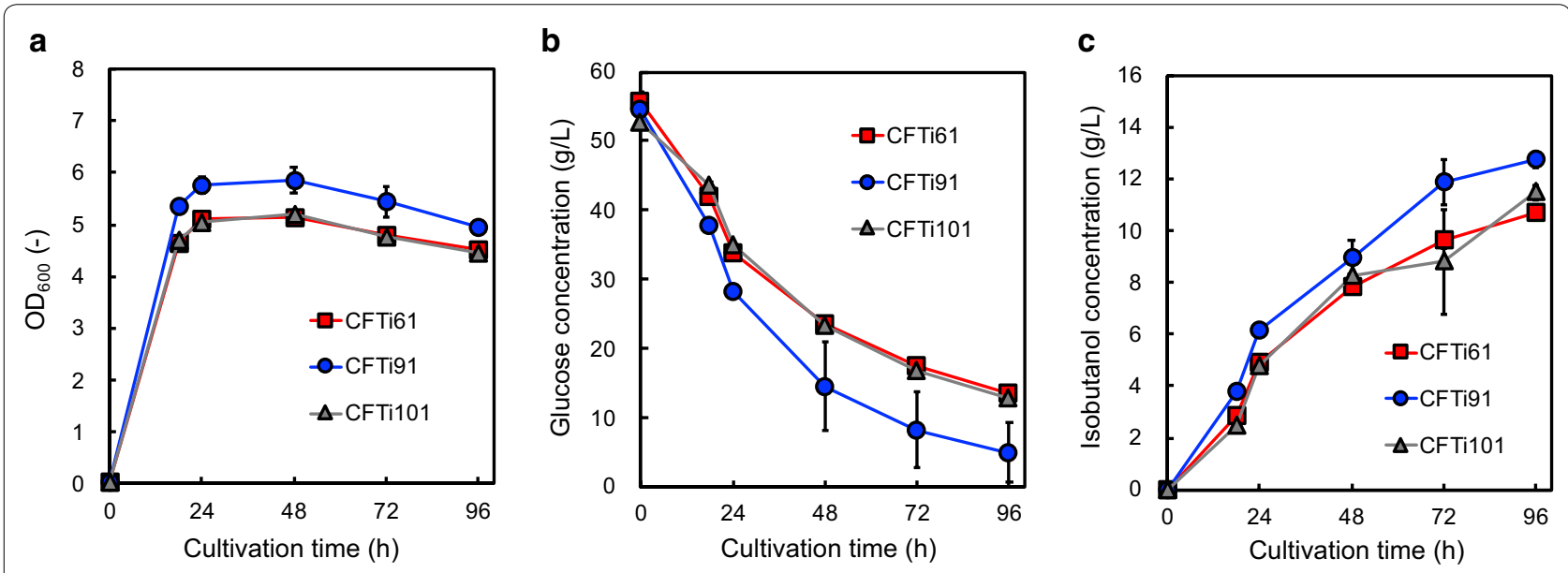

Fig. 3 CFTi61, CFTi91, and CFTi101 culture profiles. The time courses of a bacterial cell growth and $\mathbf{b}$ glucose consumption. c The amount of isobutanol produced in CFTi61 (squares), CFTi91 (circles), and CFTi101 (triangles) cultures under aerobic (until $18 \mathrm{~h}$ ) and anaerobic (after $18 \mathrm{~h}$ ) conditions. Data are presented as mean \pm standard deviation of three independent experiments

The maximal levels of cell growth, glucose consumption, and isobutanol production are shown in CFTi91 culture. Although inactivation of ldhA was effective in repressing lactate formation and improving isobutanol production, this production was decreased by removing the gene concerning acetate production (Fig. 3; Additional file 1: Fig. S3). The titer of isobutanol, yield from glucose, and rate of glucose uptake are summarized in Additional file 1: Table S3. The yield of isobutanol from glucose in CFTi91 between 18 and $96 \mathrm{~h}$ of cultivation was $0.28 \mathrm{~g} / \mathrm{g}$.
Modification of ED pathway in isobutanol-producing $E$. coli To further improve isobutanol production, we attempted to modify the ED pathway. Using a low copy number vector, the gene sets concerning the endogenous ED pathway in $E$. coli were introduced into isobutanol-producing $E$. coli transformant CFTi91 generated in the present study.

Figure 4 shows CFTi91co, CFTi91zp, and CFTi91zpee culture profiles. The highest amount of isobutanol produced was $15.0 \mathrm{~g} / \mathrm{L}$ in CFTi91zpee culture, which carries the gene set of $z w f, p g l$, edd, and eda encoding glucose-6-phosphate dehydrogenase, 6-phosphogluconolactonase, phosphogluconate dehydratase, and 

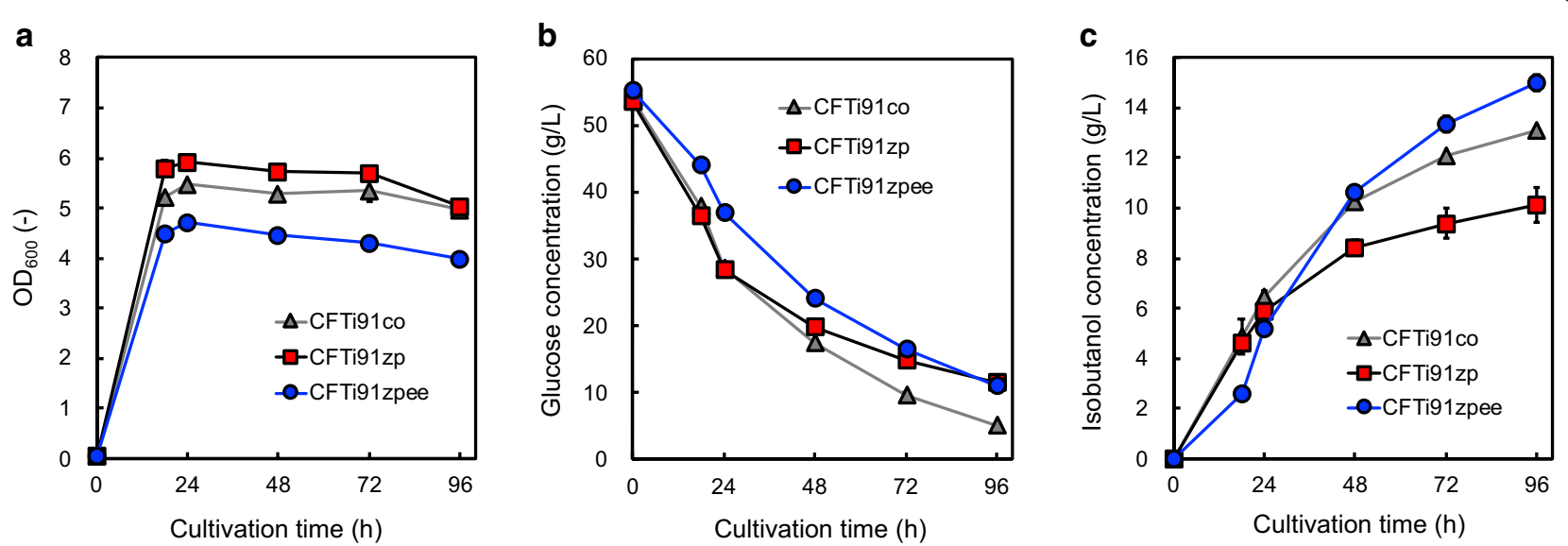

Fig. 4 CFTi91Co, CFTi91zp, and CFTi91zpee culture profiles. The time courses of $\mathbf{a}$ bacterial cell growth and $\mathbf{b}$ glucose consumption. c The amount of isobutanol produced in CFTi91co (triangles), CFTi91zp (squares), and CFTi91zpee (circles) cultures under aerobic (until $18 \mathrm{~h}$ ) and anaerobic (after $18 \mathrm{~h}$ ) conditions. Data are presented as mean \pm standard deviation of three independent experiments

Table 2 Summary of isobutanol production by each engineered $E$. coli strain

\begin{tabular}{lccll}
\hline Strain & $\mathbf{P}_{\max }(\mathbf{g} / \mathbf{L})$ & Yield $\mathbf{( g / g})$ & $\begin{array}{l}\text { Glucose } \\
\text { uptake rate } \\
\mathbf{( g / L / h})\end{array}$ & $\begin{array}{l}\text { Production } \\
\mathbf{( g / L / h})\end{array}$ \\
\hline CFTi61 & $10.7 \pm 0.1$ & $0.28 \pm 0.01$ & $0.36 \pm 0.01$ & $0.10 \pm 0.00$ \\
CFTi91 & $12.8 \pm 0.3$ & $0.28 \pm 0.02$ & $0.42 \pm 0.05$ & $0.12 \pm 0.05$ \\
CFTi101 & $11.5 \pm 0.3$ & $0.29 \pm 0.02$ & $0.39 \pm 0.01$ & $0.11 \pm 0.01$ \\
CFTi91c0 & $13.1 \pm 0.0$ & $0.25 \pm 0.01$ & $0.42 \pm 0.01$ & $0.10 \pm 0.01$ \\
CFTi91zp & $10.1 \pm 0.7$ & $0.22 \pm 0.01$ & $0.32 \pm 0.01$ & $0.07 \pm 0.01$ \\
CFTi91zpee & $15.0 \pm 0.4$ & $0.37 \pm 0.01$ & $0.42 \pm 0.00$ & $0.16 \pm 0.01$ \\
\hline
\end{tabular}

$\mathrm{P}_{\text {max }}$ the maximum amount of produced isobutanol

KHG/KDPG aldolase, respectively. Although the maximal level of cell growth and the initial rates of glucose uptake and isobutanol production until 18-h cultivation in CFTi91zpee were lower than those in CFTi91co and CFTi91zp, this production was greater in CFTi91zpee than in the others after 18-h cultivation (Fig. 4). Further, we estimated the amount of organic acids produced as byproducts. Among the CFTi91-derivative strains used, acetate and succinate production were primarily confirmed. However, their total amount was $<1.0 \mathrm{~g} / \mathrm{L}$ in each strain (Additional file 1: Fig. S4). The titer of isobutanol, yield from glucose, and rate of glucose uptake are summarized in Table 2. The yield of isobutanol from glucose in CFTi91zpee between 18 and $96 \mathrm{~h}$ cultivation was $0.37 \mathrm{~g} / \mathrm{g}$, and the final isobutanol titer was $15.0 \mathrm{~g} / \mathrm{L}$.

\section{Discussion}

Recently, microbial production using various microbes has been widely studied toward industrial application. The tools of metabolic engineering and synthetic biology could be combined to design and create our desired microorganisms [17-19]. The metabolic pathway comprises several enzymatic reactions, particularly redox reactions. Hence, it is important to design the metabolic pathway toward the compounds of interest by considering the intracellular redox balance. We adopted the ED pathway to produce isobutanol using E. coli. In the ED pathway, each $1 \mathrm{~mol}$ of NADH and NADPH is produced from $1 \mathrm{~mol}$ of glucose, whereas each $1 \mathrm{~mol}$ of NADH and $\mathrm{NADPH}$ is consumed to produce $1 \mathrm{~mol}$ of isobutanol (Fig. 1) [7]. In the present study, we generated the ED pathway-dependent $E$. coli transformants and performed isobutanol production using the derivative strains.

Several studies regarding isobutanol production using various microbes have been reported. Atsumi et al. have reported isobutanol production using $E$. coli for the first time [15]. Moreover, Corynebacterium glutamicum, B. subtilis, and Saccharomyces cerevisiae were used for isobutanol production as the host strains; however, the maximal titer and yield levels have been obtained using E. coli [20-22]. In these reports, the inactivation of genes concerning byproducts formation, direct evolution of the enzymes in the biosynthetic pathway of isobutanol, and optimization of culture conditions were performed as the general strategies to improve isobutanol production. A study on isobutanol production has demonstrated the use of partially ED pathway-dependent $E$. coli, which adopted the ED pathway derived from $Z$. mobilis [16]. However, the strain used in that study was simply introduced to the genes 
Table 3 Summary of microbial production of isobutanol

\begin{tabular}{|c|c|c|c|c|}
\hline Host & Genotype (knockout) & $P_{\max }(g / L)$ & Yield $(g / g)$ & References \\
\hline E. coli MG1655 & $\begin{array}{l}\triangle p g i \Delta g n t R \triangle g n d \\
\triangle p f B \triangle l d h A \\
\text { (Available for an endogenous ED pathway) }\end{array}$ & 15.0 & 0.37 & This study \\
\hline E. coli MG1655 & $\begin{array}{l}\triangle p f B \triangle I / d h A \triangle a c k A-p t a \\
\text { (Carrying an exogenous ED pathway) }\end{array}$ & 13.7 & 0.31 & [16] \\
\hline E. coli BW25113 & $\begin{array}{l}\triangle a d h E \triangle l d h A \triangle f r d B C \\
\triangle f n r \Delta p t a \triangle p f l B \\
\text { (EM pathway) }\end{array}$ & 22 & 0.35 & [15] \\
\hline C. glutamicum ATCC13032 & $\begin{array}{l}\triangle a c e E \Delta p q o \triangle i l v E \Delta l d h A \\
\Delta m d h \text { (EM pathway) }\end{array}$ & 13 & 0.2 & [23] \\
\hline B. subtilis & $\Delta / d h A$ (EM pathway) & 2.62 & N.E. & [24] \\
\hline
\end{tabular}

$\mathrm{NE}$, not estimated

concerning the ED pathway, and the redox balance in the biosynthesis pathway involved in converting glucose to isobutanol was not completely considered. The yield of isobutanol from glucose $(0.31 \mathrm{~g} / \mathrm{g})$ was lower than that in the case using the EM pathway and has the potential for further improvements [16, 20].

In the present study, we constructed the ED pathway-dependent $E$. coli transformants by inactivating the negative repressor of the ED pathway and the genes concerning the initial reaction of EM and PPP pathway (Fig. 1). First, we inactivated pgi to repress the carbon flux to the EM pathway. The rates of glucose uptake and isobutanol production in CFTi31 were decreased, and acetate production as the byproduct was lower than that of CFTi21, which is completely dependent on the EM pathway (Additional file 1: Fig. S2). CFTi21 produced isobutanol mainly in growth phase, whereas did not in stationary phase (Fig. 2). This may be because CFTi21 produced a large amount of acetate along with isobutanol and decrease of $\mathrm{pH}$ caused lysis of bacterial cell. Although $g n t R$ was disrupted after removing $p g i$ to activate the ED pathway, the amount of isobutanol production in CFTi41 was drastically decreased compared with that in CFTi21 or CFTi31 (Fig. 2). This could be attributed to the cell growth of CFTi41, which was greater than that of other strains used in this section; moreover, the PPP pathway and TCA cycle, which are important for cell growth may be activated in CFTi41, whose pgi and $g n t R$ were inactivated (Fig. 2). Finally, gnd catalyzing the third reaction of the PPP pathway was inactivated. Although the cell growth of CFTi51 was decreased compared with that of CFTi41, the isobutanol production was almost threefold higher (Fig. 2). This could be attributed to the repression of carbon leak to the PPP pathway and stimulation of the carbon flux to isobutanol from glucose through the ED pathway. The maximal titer and yield of isobutanol were recorded in the ED pathway-dependent CFTi51.
Thereafter, we changed the culture condition to prevent volatilization of isobutanol. Although culturing was initiated in aerobic condition, test tubes used here were screw-capped after $18 \mathrm{~h}$ of cultivation. To prevent the formation of organic acids associated with the change in culture condition, we inactivated genes concerning organic acid formation. Although the disruptions of $p f l B$ and $l d h A$ concerning formate and lactate formation, respectively, were effective to improve isobutanol production, the inactivation of pta gene resulted in decreasing isobutanol production (Fig. 3). However, the amount of acetate produced in CFTi91 was only $250 \mathrm{mg} / \mathrm{L}$, and the carbon from glucose would be used for isobutanol production (Additional file 1: Fig. S3). Here, we speculated the reason why the deletion of pta gene had a negative effect to isobutanol production, despite of lacking anaerobic source of acetyl-CoA which is a substrate of Pta. As shown in Additional file 1: Fig. S3A, the isobutanol-producing strain whose pta gene was deleted drastically decreased acetate production. We assumed that a small amount of produced acetate until $24 \mathrm{~h}$ cultivation (aerobic or micro-aerobic condition) was one of key factor in the isobutanol production using our created strain. The energy balance optimized by deleting both pflB and ldhA genes might be disturbed by omitting the gene concerning acetate production.

To further improve isobutanol production, we attempted to modify the endogenous ED pathway in $E$. coli. Although the initial isobutanol production and the maximal cell growth were decreased by introducing 4 genes constructing the ED pathway, the final titer of isobutanol and yield from glucose were encouraged (Fig. 4; Table 2). In the optimized strain CFTi91zpee as well as in CFTi91, acetate production was repressed, whereas succinate production was relatively increased (Additional file 1: Fig. S4). In the present study, the maximal yield of isobutanol from glucose reached $0.37 \mathrm{~g} / \mathrm{g}$, which was 
higher than that in a previous report using partially ED pathway-dependent strain for isobutanol production and $90 \%$ of the maximal theoretical yield $(0.41 \mathrm{~g} / \mathrm{g})$ [16]. In addition, it would be comparable with other reports on isobutanol production using various host strains (Table 3 ) [20]. These results indicate that the redox-balanced metabolic design is one of the significant factors in microbial production. Although we reconstructed metabolic pathway suitable for isobutanol production from the aspect of the redox balance, $E$. coli is known to have the endogenous transhydrogenase encoded in pntAB concerning the conversion of NADH into NADPH which is a candidate reaction to acquire NADPH [21]. In the previous report about isobutanol production, the coexpression of PntAB and NAD kinase contributed to encouraging isobutanol production [22]. This result would mean that those two enzymes needed to be overexpressed to earn the enough activity of converting NADH to NADPH, which is one of essential cofactors for isobutanol biosynthetic pathway.

In the experiment keeping under anaerobic condition after $18 \mathrm{~h}$ cultivation, succinate production was confirmed (Additional file 1: Figs. S3C and S4B). the isobutanol-producing strain used in the study lacked the intracellular $\mathrm{CO}_{2}$ sources the gene $p f l B$, oxidative TCA cycle, and the oxidative pentose phosphate pathway, however; $2 \mathrm{~mol}$ of $\mathrm{CO}_{2}$ was produced in the exogenous biosynthetic pathway of isobutanol (Fig. 1). Succinate production would be attributed to the activation of phosphoenolpyruvate (PEP) carboxylase which produces oxaloacetate from PEP and $\mathrm{CO}_{2}$. The produced oxaloacetate might be converted into succinate via the reductive TCA cycle to prevent overflowing the intracellular excess energy.

In conclusion, for effective isobutanol production, we generated ED pathway-dependent E. coli strain, which produced $11.8 \mathrm{~g} / \mathrm{L}$ of isobutanol, and the yield from glucose was $0.24 \mathrm{~g} / \mathrm{g}$ under aerobic condition. By inactivating genes concerning organic acids and modifying the endogenous ED pathway, isobutanol production was increased, and the titer and yield from glucose were $15.0 \mathrm{~g} / \mathrm{L}$ and $0.37 \mathrm{~g} / \mathrm{g}$, respectively. The optimization of culture conditions and the collection of volatilized isobutanol are expected to further improve isobutanol production.

\section{Methods}

\section{Strains and plasmid construction}

The strains and plasmids used in the present study are listed in Table 1 and Additional file 1: Table S1. E. coli NovaBlue competent cells (Novagen, Cambridge, MA, USA) were used for gene cloning. Polymerase chain reaction (PCR) was performed using the KOD FX Neo DNA polymerase (Toyobo, Osaka, Japan), and the primer pairs are listed in Additional file 1: Table S1. Each gene was assembled with the respective plasmid using Gibson Assembly (New England Biolabs, Ipswich, MA, USA). The construction of plasmids used in this study is described in Table 1. The plasmids were transformed into bacterial strains using the Gene Pulser II (Bio-Rad, Hercules, CA, USA). Wherever applicable, $100 \mu \mathrm{g} / \mathrm{mL}$ ampicillin, $50 \mu \mathrm{g} / \mathrm{mL}$ kanamycin, and $15 \mu \mathrm{g} / \mathrm{mL}$ chloramphenicol were added to the media for selection purpose. All the transformants constructed in this study are listed in Table 1.

pZA23trc was constructed as follows: The nucleotide sequence of $\operatorname{trc}$ promoter and pZA23 without the promoter region were amplified by PCR using pTrcHis B and pZA23MCS as the templates with the primer pairs inv_pZA23_no_prom_f and inv_pZA23_no_prom_r. The amplified fragments were fused with each other, and the resulting plasmid was designated as pZA23trc. pZka and pTka were constructed as follows: the synthetic gene fragments of kivd and adhA were directly cloned into the KpnI and HindIII sites of pZE12MCS, respectively, and the resulting plasmid was designated as pZka. Subsequently, the gene set of kivd and adhA and the plasmid fragment of $\mathrm{pTrcHis} B$ were amplified by PCR using pZka and pTrcHis $B$ as the template with the primer pair kivd_ptrc_f and adhA_ptrc_r and inv_ptrc_f and inv_ptrc_r, respectively. The amplified fragments were fused with each other, and the resulting plasmid was designated as pTka. p23S and p23SCD were constructed as follows: alsS (NP_391482.2) and pZA23trc fragment were amplified by PCR using $B$. subtilis genomic DNA and pZA23trc as the template with the primer pairs alsS_ptrc_f and alsS_ptrc_r and inv_ptrc_f and inv_ptrc_r, respectively. The amplified fragments were fused together, and the resulting plasmid was designated as p23S. Thereafter, the gene set of ilvC (NP_418222.1) and ilvD (YP_026248.1) was amplified by PCR using $E$. coli K-12 MG1655 genomic DNA as the template and the primer pair ilvCD_ptrc_sacI _f and ilvCD_ptrc_sacI_r. The amplified fragment was cloned into the SacI site of $\mathrm{p} 23 \mathrm{~S}$, and the resulting plasmid was designated as p23SCD. pSp, pSzp, and pSzpee were constructed as follows: pgl (NP_415288.1) and pSAK fragment were amplified by PCR using E. coli K-12 MG1655 genomic DNA and pSAK as the template and the primer pairs pgl_pZ_ hindIII_f and pgl_pZ_hindIII_r and inv_pZ_hindIII_f and inv_pZ_hindIII_r, respectively. The amplified fragments were fused together, and the resulting plasmid was designated as pSp. $z w f$ (NP_416366.1) was amplified by PCR using E. coli K-12 MG1655 genomic DNA as the template with the primer pair zwf_pZ_kpnI_f and zwf_pZ_kpnI _r. The amplified fragment was cloned into the KpnI site of $\mathrm{pSp}$, and the resulting plasmid was designated as pSzp. 
edd (NP_416365.1) and eda (NP_416364.1) were amplified by PCR using E. coli K-12 MG1655 genomic DNA as the template with the primer pairs edd_pZ_bamHI_f and edd _r and eda_f and eda_pZ_bamHI_r, respectively. The gene set of $e d d$ and $e d a$ were amplified by PCR using these amplified fragments of edd and eda as the templates with the primer pair i edd_pZ_bamHI_f and eda pZ_bamHI_r. The amplified fragment was cloned into the BamHI site of pSzp, and the resulting plasmid was designated as pSzpee. Plasmid maps used in this study are shown in Additional file 1: Fig. S5.

\section{Deletion of chromosomal genes}

The primers used for the deletion of chromosomal genes are listed in Additional file 1: Table S1. E. coli K-12 MG1655 (ATCC700926) was used as the parent strain. Using the Quick and Easy E. coli Gene Deletion Kit (Funakoshi, Tokyo, Japan), each gene was deleted according to the manufacturer's protocol. The gene fragments for deletion and recombination were introduced into each strain using the Gene Pulser II. Other fragments used to inactivate the respective genes were amplified by PCR using the FRT-PGK-gp2-neo-FRT template DNA from the Quick and Easy E. coli Gene Deletion Kit with the appropriate primers, as listed in Additional file 1: Table S1.

\section{Culture conditions}

The M9Y medium was used for isobutanol production in $5-\mathrm{mL}$ test tube-scale cultures. M9Y minimal medium contains (per liter): glucose, 20 g; yeast extract, 5 g; NaCl, 0.5 g; $\mathrm{Na}_{2} \mathrm{HPO}_{4} \cdot 12 \mathrm{H}_{2} \mathrm{O}, 17.1$ g; $\mathrm{KH}_{2} \mathrm{PO}_{4}, 3$ g; $\mathrm{NH}_{4} \mathrm{Cl}, 2$ g; $\mathrm{MgSO}_{4} \cdot 7 \mathrm{H}_{2} \mathrm{O}, 246 \mathrm{mg} ; \mathrm{CaCl}_{2} \cdot 2 \mathrm{H}_{2} \mathrm{O}, 14.7 \mathrm{mg}$; $\mathrm{FeSO}_{4} \cdot 7 \mathrm{H}_{2} \mathrm{O}, 2.78 \mathrm{mg}$; thiamine hydrochloride, $10 \mathrm{mg}$. Wherever applicable, ampicillin, kanamycin, and/or chloramphenicol were added to the medium at a final concentration of 100,50 , and $15 \mu \mathrm{g} / \mathrm{mL}$, respectively. Each preculture was seeded to $5 \mathrm{~mL}$ of M9Y medium in a test tube at an initial $\mathrm{OD}_{600}$ of 0.05 . Test tube-scale cultures were incubated at $37{ }^{\circ} \mathrm{C}$ in a shaker at $220 \mathrm{rpm}$. IPTG $(0.1 \mathrm{mM})$ was added to the culture medium at $\mathrm{OD}_{600}$ of 0.5 , following which test tube-scale cultures were incubated at $30{ }^{\circ} \mathrm{C}$ in a shaker at $220 \mathrm{rpm}$. The trace experiments with $\left[1-{ }^{13} \mathrm{C}\right]$ glucose were performed as below. The M9 medium was used in 5-mL test tube-scale cultures. M9Y minimal medium contains (per liter): $\left[1-{ }^{13} \mathrm{C}\right]$ glucose, $20 \mathrm{~g} ; \mathrm{NaCl}, 0.5 \mathrm{~g} ; \mathrm{Na}_{2} \mathrm{HPO}_{4} \cdot 12 \mathrm{H}_{2} \mathrm{O}, 17.1 \mathrm{~g} ; \mathrm{KH}_{2} \mathrm{PO}_{4}$, 3 g; $\mathrm{NH}_{4} \mathrm{Cl}, 2$ g; $\mathrm{MgSO}_{4} \cdot 7 \mathrm{H}_{2} \mathrm{O}, 246 \mathrm{mg} ; \mathrm{CaCl}_{2} \cdot 2 \mathrm{H}_{2} \mathrm{O}$, $14.7 \mathrm{mg}$; $\mathrm{FeSO}_{4} \cdot 7 \mathrm{H}_{2} \mathrm{O}, 2.78 \mathrm{mg}$; thiamine hydrochloride, $10 \mathrm{mg}$. Other culture conditions were the same as isobutanol-production experiments.

\section{Analytical methods}

Cell growth was monitored by measuring $\mathrm{OD}_{600}$ with a UVmini-1240 spectrophotometer (Shimadzu, Kyoto, Japan). The concentration of glucose in the culture supernatant was measured using the Glucose CII test (Wako, Kyoto, Japan) according to the manufacturer's protocol.

Gas chromatography-mass spectrometry (GC-MS) was performed using a GCMS-QP2010 Ultra instrument (Shimadzu) equipped with a DB-FFAP column (60 m, 0.25- $\mathrm{mm}$ internal diameter, $0.5-\mathrm{mm}$ film thickness; Agilent). Helium was used as the carrier gas to maintain a flow rate of $2.1 \mathrm{~mL} / \mathrm{min}$. The injection volume was $1 \mu \mathrm{L}$ with a split ratio of 1:10. The amount of isobutanol was quantified as follows: the oven temperature was initially maintained at $40{ }^{\circ} \mathrm{C}$ for $1 \mathrm{~min}$, following which it was gradually raised to $195^{\circ} \mathrm{C}$ at $10{ }^{\circ} \mathrm{C} / \mathrm{min}$ and further to $250{ }^{\circ} \mathrm{C}$ at $120^{\circ} \mathrm{C} / \mathrm{min}$ before finally being maintained at $250{ }^{\circ} \mathrm{C}$ for $3 \mathrm{~min}$. The total running time was $20 \mathrm{~min}$. The other settings included maintaining the interface temperature at $250{ }^{\circ} \mathrm{C}$, ion-source temperature at $200{ }^{\circ} \mathrm{C}$, and electron impact ionization at $70 \mathrm{eV}$.

Further, the concentration of organic acids was determined in the culture supernatants that were separated from the culture broth by centrifugation at $21,880 \times g$ for $20 \mathrm{~min}$. The concentrations of organic acids as byproducts were determined using an organic acid analysis system (Shimadzu) consisting of an HPLC instrument equipped with a Shim-pack SPR-H column. The column was operated at $48{ }^{\circ} \mathrm{C}$ with a flow rate of $0.8 \mathrm{~mL} \mathrm{~min}$. CDD-10A was used as the detector. $p$-Toluene sulphonic acid $(5 \mathrm{mM})$ was used as the mobile phase, and 20-mM bis-Tris containing 5-mM $p$-toluene sulphonic acid and $100-\mu \mathrm{M}$ ethylenediaminetetraacetic acid was mixed immediately before the detection to enhance the sensitivity.

\section{Additional file}

Additional file 1. Additional files and tables.

\section{Abbreviations}

EM pathway: Embden-Meyerhof pathway; ED pathway: Entner-Doudoroff pathway; ATP: adenosine triphosphate; NADH: reduced nicotinamide adenine dinucleotide; NADPH: reduced nicotinamide adenine dinucleotide phosphate; PPP: pentose phosphate pathway; PCR: polymerase chain reaction; GC-MS: gas chromatography-mass spectrometry; acetyl-CoA: acetyl coenzyme A.

\section{Acknowledgements}

The authors would like to thank Enago (http://www.enago.jp) for the English language review. 


\section{Authors' contributions}

SN, MA, and TS designed the work. SN, YM, SO, and TS performed the experiments. SN, YM, MA, and TS discussed the data. AK encouraged this project. SN and TS wrote the paper. All authors read and approved the final manuscript.

\section{Funding}

This work has been supported by the RIKEN Center for Sustainable Resource Science, and the commission for Development of Artificial Gene Synthesis Technology for Creating Innovative Biomaterial from the Ministry of Economy, Trade and Industry (METI), Japan.

\section{Availability of data and materials}

The datasets supporting this work are included in the manuscript and additional file.

\section{Ethics approval and consent to participate}

Not applicable.

\section{Consent for publication}

Not applicable.

\section{Competing interests}

The authors declare that they have no competing interests.

\section{Author details}

${ }^{1}$ Center for Sustainable Resource Science, RIKEN, 1-7-22, Suehiro-cho, Tsurumi-ku, Yokohama, Kanagawa 230-0045, Japan. ${ }^{2}$ Department of Chemical Science and Engineering, Graduate School of Engineering, Kobe University, 1-1 Rokkodai, Nada, Kobe 657-8501, Japan. ${ }^{3}$ Graduate School of Medicine, Kyoto University, 54 Kawahara-cho, Syogoin, Sakyo-ku, Kyoto 606-8507, Japan.

Received: 10 March 2019 Accepted: 2 July 2019

Published online: 18 July 2019

\section{References}

1. Becker J, Wittmann C. From systems biology to metabolically engineered cells-an omics perspective on the development of industrial microbes. Curr Opin Microbiol. 2018;45:180-8.

2. Ekas H, Deaner M, Alper HS. Recent advancements in fungal-derived fuel and chemical production and commercialization. Curr Opin Biotechnol. 2018;57:1-9.

3. Noda S, Kondo A. Recent advances in microbial production of aromatic chemicals and derivatives. Trends Biotechnol. 2017;35:785-96.

4. Liao JC, Mi L, Pontrelli S, Luo S. Fuelling the future: microbial engineering for the production of sustainable biofuels. Nat Rev Microbiol. 2016;14:288-304.

5. Choi S, Song CW, Shin JH, Lee SY. Biorefineries for the production of top building block chemicals and their derivatives. Metab Eng. 2015:28:223-39.

6. Nielsen J, Larsson C, van Maris A, Pronk J. Metabolic engineering of yeast for production of fuels and chemicals. Curr Opin Biotechnol. 2013;24:398-404

7. Klingner A, Bartsch A, Dogs M, Wagner-Döbler I, et al. Large-scale $13 C$ flux profiling reveals conservation of the Entner-Doudoroff pathway as a glycolytic strategy among marine bacteria that use glucose. Appl Environ Microbiol. 2015;81:2408-22.

8. $\mathrm{Ng} \mathrm{CY}$, Farasat I, Maranas CD, Salis HM. Rational design of a synthetic Entner-Doudoroff pathway for improved and controllable NADPH regeneration. Metab Eng. 2015;29:86-96.

9. Zhao N, Bai Y, Liu CG, Zhao XQ, Xu JF, Bai FW. Flocculating Zymomonas mobilis is a promising host to be engineered for fuel ethanol production from lignocellulosic biomass. Biotechnol J. 2014:9:362-71.
10. Long CP, Gonzalez JE, Feist AM, Palsson BO, Antoneiwicz MR, et al. Dissecting the genetic and metabolic mechanisms of adaptation to the knockout of a major metabolic enzyme in Escherichia coli. Proc Natl Acad Sci USA. 2018;115:222-7.

11. Chen X, Schreiber K, Appel J, Makowka A, Fahnrich B, Roettger M, et al. The Entner-Doudoroff pathway is an overlooked glycolytic route in cyanobacteria and plants. Proc Natl Acad Sci USA. 2016;113:5441-6.

12. Zhang Y, Lin Z, Liu Q, Li Y, Wang Z, Ma H, et al. Engineering of SerineDeamination pathway, Entner-Doudoroff pathway and pyruvate dehydrogenase complex to improve poly(3-hydroxybutyrate) production in Escherichia coli. Microb Cell Fact. 2014;13:172-82.

13. Hong SH, Park SJ, Moon SY, Park JP, Lee SY. In silico prediction and validation of the importance of the Entner-Doudoroff pathway in poly(3hydroxybutyrate) production by metabolically engineered Escherichia coli. Biotechnol Bioeng. 2003;83:854-63.

14. Atsumi S, Wu TY, Eckl EM, Hawkins SD, Buelter T, Liao JC. Engineering the isobutanol biosynthetic pathway in Escherichia coli by comparison of three aldehyde reductase/alcohol dehydrogenase genes. Appl Microbiol Biotechnol. 2010;85:651-7.

15. Atsumi S, Hanai T, Liao JC. Non-fermentative pathways for synthesis of branched-chain higher alcohols as biofuels. Nature. 2008;451:86-9.

16. Liang S, Chen H, Liu J, Wen J. Rational design of a synthetic Entner-Doudoroff pathway for enhancing glucose transformation to isobutanol in Escherichia coli. J Ind Microbiol Biotechnol. 2018;45:187-99.

17. Wu G, Yan Q, Jones JA, Tang YJ, Fong SS, Koffas MAG. Metabolic burden: cornerstones in synthetic biology and metabolic engineering applications. Trends Biotechnol. 2016;34:652-64.

18. Lee SY, Kim HU. Systems strategies for developing industrial microbial strains. Nat Biotechnol. 2015;33:1061-72.

19. Yadav VG, De Mey M, Lim CG, Ajikumar PK, Stephanopoulos G. The future of metabolic engineering and synthetic biology: towards a systematic practice. Metab Eng. 2012;14:233-41.

20. Choi YJ, Lee J, Jang YS, Lee SY. Metabolic engineering of microorganisms for the production of higher alcohols. MBio. 2014;5:e01524.

21. Akinterinwa O, Cirino PC. Anaerobic obligatory xylitol production in Escherichia coli strains devoid of native fermentation pathways. Appl Environ Microbiol. 2010;77:706-9.

22. Shi A, Zhu X, Lu J, Zhang X, Ma Y. Activating transhydrogenase and NAD kinase in combination for improving isobutanol production. Metab Eng 2012;16:1-10.

23. Blombach B, Riester T, Wieschalka S, Ziert C, Youn JW, Wendisch VF, et al. Corynebacterium glutamicum tailored for efficient isobutanol production. Appl Environ Microbiol. 2011;77:3300-10.

24. Li S, Wen J, Jia X. Engineering Bacillus subtilis for isobutanol production by heterologous Ehrlich pathway construction and the biosynthetic 2-ketoisovalerate precursor pathway overexpression. Appl Microbiol Biotechnol. 2011:91:577-89.

\section{Publisher's Note}

Springer Nature remains neutral with regard to jurisdictional claims in published maps and institutional affiliations.

Ready to submit your research? Choose BMC and benefit from

- fast, convenient online submission

- thorough peer review by experienced researchers in your field

- rapid publication on acceptance

- support for research data, including large and complex data types

- gold Open Access which fosters wider collaboration and increased citations

- maximum visibility for your research: over $100 \mathrm{M}$ website views per year

At $\mathrm{BMC}$, research is always in progress.

Learn more biomedcentral.com/submissions 